\title{
Does exercise reduce all-cancer death rates?
}

\author{
Roy J. Shephard \\ School of Physical and Health Education and Department of Preventive Medicine and Biostatistics, Faculty of \\ Medicine, University of Toronto, Canada
}

\begin{abstract}
A reanalysis is made of earlier data relating to initial physical fitness and the likelihood of death from all forms of cancer. It is argued that the original analysis may have been biased by an association between initial fitness and other health habits, particularly cigarette smoking. The association with fitness status remains after reanalysis of the data on the assumption that current smoking leads to a uniform doubling of the risk of cancer death, but the effect is weaker than previously reported. There remains some potential bias, in that the quantity of current smoking may have been linked to fitness status. Some $55 \%$ of deaths were untraced, but it is argued that any socioeconomic or other bias from this cause is likely to account for the association between cancer risk and low fitness status. Any reduction of cancer risk is associated with the change from an extremely sedendary to a moderately sedentary lifestyle. It thus cannot be explained in terms of the mechanisms previously invoked to explain low risks of colonic and reproductive cancers in endurance athletes.
\end{abstract}

Keywords: Preventive medicine, exercise, neoplasms, carcinoma, smoking, physical activity

Recent reviews ${ }^{1,2}$ have noted an association between high levels of occupational or recreational physical activity and protection against certain forms of cancer, including neoplasms of the colon and the female reproductive tract. The most dramatic effect is presented in a recent paper by Blair and associates ${ }^{3}$ which analysed data from 10224 men and 3120 women attending a preventive medical examination at the Cooper Clinic in Dallas. The primary purpose of the study was to relate all-cause mortality over an average follow-up of 8 years to initial fitness levels as assessed by a maximal treadmill test. From detailed analysis of the findings it was concluded that physical fitness 'appear(s) to delay all-cause mortality primarily due to lowered rates of cardiovascular disease and cancer'.

The gradient of all-cause mortality with fitness levels was said to persist when the data were adjusted for smoking habits. Nevertheless, there remains a possibility that all or part of the apparently very steep fitness-related gradient within the cancer deaths was due to an association between smoking habits and exercise behaviour ${ }^{4}$. The data have thus been reanalysed to test this possibility.

Address for correspondence: Professor Roy J. Shephard, Director School of Physical and Health Education, 320 Huron Street,

Toronto, Ontario M5S 1A1, Canada

(C) 1992 Butterworth-Heinemann Ltd 0306-3674/92/020125-04

\section{Subjects and methods}

The data that are here reanalysed were collected by Blair et al. ${ }^{3}$ on 10224 men and 3120 women who received a preventive medical examination, including an exercise stress test, at the Cooper Clinic in Dallas between 1970 and 1981. Criteria for inclusion were residence in the USA and attainment of at least $85 \%$ of their age-predicted maximal heart rate during the test.

The treadmill speed was initially set at $88 \mathrm{~m} \mathrm{~min}^{-1}$, $0 \%$ grade, with a $2 \%$ grade in the second minute, and a $1 \%$ increment of grade in each subsequent minute to $25 \mathrm{~min}$. Thereafter, a grade of $25 \%$ was maintained, but speed was increased by $5.4 \mathrm{~m} \mathrm{~min}^{-1}$ until the patient was exhausted. Fitness was gauged from the treadmill time, sex and age, results being classified as low fitness (lowest quintile), medium fitness (second and third quintiles) and high (fourth and fifth quintiles).

Cigarette smoking was determined by questionnaire. Patients who reported smoking at present or within the last 2 years were classed as 'current smokers'.

The average follow-up period was just over 8 years, with vital status being ascertained in $95 \%$ of participants over a total of 110482 person-years; we have assumed a similar average follow-up period for the two sexes, i.e. 84650 person-years of experience for the men and 25832 person-years for the women. There were 283 deaths in this sample, classified in accordance with the International Classification of Diseases.

\section{Results}

\section{Unadjusted data}

Cancer of all types (ICD-9, 140-208) accounted for $64 / 240$ deaths $(26.7 \%)$ in the men, and $18 / 43$ deaths $(41.9 \%)$ in the women. There was a steep fitnessrelated gradient for the overall cancer mortality rate, from a rate per 10000 person-years of 20.3 in the least fit male quintile to 4.7 in the men of the two most fit quintiles. Moreover, this gradient was even steeper in women, with rates of 16.3 and 1.0 per 10000 person-years in the corresponding fitness quintiles.

\section{Reanalysis of data}

Men

Looking first at data for the men, classified by smoking habits and fitness category, the matrix 
showing the total number of all-cause deaths $(\mathrm{T})$ and the risk of all-cause mortality per 10000 individuals $(\mathrm{R})$ is shown in Table 1.

The corresponding sample size in person-years can be estimated from the number of deaths per cell (T) and the risk (R). For example, in high-fitness subjects who never smoked, there were 32 deaths and a risk of 16 per 10000 , giving a cell size of 20000 . The resulting total person-year matrix is shown in Table 2.

From the population size and the age-adjusted relative risks shown by Blair et al. ${ }^{3}$ (in their Table 4), the total cancer deaths can be calculated for each of the three fitness categories. The total somewhat exceeds the reported 64 cancer deaths actually observed, but by scaling the mortality rate for each of the three fitness categories downwards, there would be totals of 26,22 and 16 cases, respectively, in the three fitness categories. The risk of cancer death is at least doubled by smoking, and the smoking cells should thus be attributed twice as many cases as would be predicted from their population size. The corresponding matrix for the 64 cancer deaths may thus be approximated by the non-integer values shown in Table 3.

If the excess of smoking-related cancer deaths $(50 \%$ of the first row in Table 3) is excluded from each of the three fitness categories, the matrix can be revised as in Table 4.

Table 1. Mortality data for men classified by smoking habits and fitness category

\begin{tabular}{|c|c|c|c|c|}
\hline \multirow{3}{*}{ Smoking habit } & \multicolumn{3}{|c|}{ Fitness category } & \multirow{3}{*}{ Total $(T)$} \\
\hline & \multirow{2}{*}{$\frac{\text { Low }}{T R}$} & \multirow{2}{*}{$\frac{\text { Medium }}{T R}$} & \multirow{2}{*}{$\begin{array}{l}\text { High } \\
T R\end{array}$} & \\
\hline & & & & \\
\hline $\begin{array}{l}\text { Current smoker } \\
\text { Ex-smoker } \\
\text { Never smoked }\end{array}$ & $\begin{array}{ll}49 & 80 \\
10 & 44 \\
16 & 26\end{array}$ & $\begin{array}{ll}51 & 47 \\
10 & 13 \\
26 & 16\end{array}$ & $\begin{array}{ll}29 & 41 \\
17 & 19 \\
32 & 16\end{array}$ & $\begin{aligned} 129 & (54) \\
37 & (15) \\
74 & (31)\end{aligned}$ \\
\hline Total no. of deaths & & & & $240(100)$ \\
\hline
\end{tabular}

Values in parentheses are percentages. $T=$ no. of deaths per cell; $R=$ risk of death per 10000 subjects

Table 2. Total person-year matrix classified by smoking habits and fitness category (male subjects)

\begin{tabular}{lrrrr}
\hline & \multicolumn{3}{c}{ Fitness category } & \\
\cline { 2 - 4 } Smoking habit & Low & Medium & High & Total \\
\hline Current smoker & 6125 & 10851 & 7073 & 24049 \\
Ex-smoker & 2272 & 7692 & 8947 & 18911 \\
Never smoked & 6154 & 16250 & 20000 & 42404 \\
Total & 14551 & 34793 & 36020 & 85364 \\
\hline
\end{tabular}

As a result of slight rounding errors, the total is 85364 rather than 84650 person-years
Table 3. Likely number of cancer deaths in each of cells, assuming smoking doubles risk for given fitness status (male subjects)

\begin{tabular}{lrrrr}
\hline & \multicolumn{3}{c}{ Fitness category } & \\
\cline { 2 - 4 } & Low & Medium & High & Total \\
\hline Smoking habit & 15.4 & 10.5 & 5.3 & $31(48)$ \\
Current smoker & 2.8 & 3.7 & 3.3 & $10(16)$ \\
Ex-smoker & 7.8 & 7.8 & 7.4 & $23(36)$ \\
Never smoked & & & & $64(100)$ \\
Total & & & & \\
\hline
\end{tabular}

Values in parentheses are percentages of total deaths

Table 4. Recalculation of Table 3 excluding excess deaths due to smoking

Fitness category

\begin{tabular}{lrrrr}
\cline { 2 - 4 } Smoking habit & Low & Medium & High & Total \\
\hline Current smoker & 7.7 & 5.3 & 2.5 & 15.5 \\
Ex-smoker & 2.8 & 3.7 & 3.3 & 9.8 \\
Never smoked & 7.8 & 7.8 & 7.4 & 23.0 \\
Total & 18.3 & 16.8 & 13.2 & 48.3 \\
\hline
\end{tabular}

Table 5. All-cause mortality data for women classified by smoking habits and fitness status

\begin{tabular}{lcccr}
\hline & \multicolumn{3}{c}{ Fitness category } & \\
\cline { 2 - 4 } Smoking habit & Low & Medium & High & Total \\
\hline Current smoker & 8 & 6 & 3 & $17(39.5)$ \\
Ex-smoker & 4 & 2 & 3 & $9(21.0)$ \\
Never smoked & 6 & 8 & 3 & $17(39.5)$ \\
\hline
\end{tabular}

Values in parentheses are percentages of total deaths

If the small impact upon overall sample size of the 15.5 smoking-related cancers that we have excluded from our column totals is ignored, the risk of cancers unrelated to smoking per 10000 person-years in each of the three fitness categories may be approximated by relating the column totals from the revised matrix to the corresponding total person-years of experience, as calculated above. The cancer risk per 10000 person-years in each of the fitness categories is then as follows: low, 12.6; medium, 4.8; high, 3.7.

\section{Women}

Analogous calculations can be performed when reanalysing the data for women, although here the sample size is smaller and the numbers are correspondingly less reliable. Thus, the initial all-cause death matrix is as shown in Table 5.

The corresponding sample size in person-years is shown in Table 6.

Again allowing for a doubling of cancer deaths in the smokers, the corresponding matrix for all cancer deaths may be approximated as shown in Table 7. 
Excluding the excess of smoking-related cancer deaths from the first row, the revised matrix for the three fitness categories is given in Table 8.

The relative risk of cancer per 10000 person-years for the different fitness categories is then as follows: low, 11.5; medium, 7.6; high, 0.9 .

Thus, in both men and women, there is apparently some residual association between fitness levels and overall cancer rate after elimination of the likely effect of smoking, with age-adjusted risk ratios as shown in Table 9. In other words, the risk of cancer (other than

Table 6. Total person-year matrix classified by smoking habits and fitness category (female subjects)

\begin{tabular}{lrrrr}
\hline & \multicolumn{3}{c}{ Fitness category } & \\
\cline { 2 - 4 } Smoking habit & Low & Medium & High & Total \\
& & & & \\
\hline Current smoker & 1403 & 2609 & 1364 & 5376 \\
Ex-smoker & 800 & 1818 & 3000 & 5618 \\
Never smoked & 2400 & 6154 & 6000 & 14554 \\
Total & 4603 & 10581 & 10364 & 25548 \\
\hline
\end{tabular}

Table 7. Likely number of cancer deaths in each of cells, assuming smoking doubles risk for given fitness status (female subjects)

\begin{tabular}{lcccc}
\hline & \multicolumn{3}{c}{ Fitness category } & \\
\cline { 2 - 4 } Smoking habit & Low & Medium & High & \multirow{2}{*}{ Total } \\
\hline Current smoker & 3.3 & 4.0 & 0.2 & $7.5(41.7)$ \\
Ex-smoker & 0.9 & 1.4 & 0.3 & $2.6(14.4)$ \\
Never smoked & 2.8 & 4.6 & 0.5 & $7.9(43.9)$ \\
\hline
\end{tabular}

Values in parentheses are percentages of total deaths

Table 8. Recalculation of Table 7 excluding excess deaths due to smoking

\begin{tabular}{lcccc}
\hline & \multicolumn{3}{c}{ Fitness category } & \\
\cline { 2 - 4 } Smoking habit & Low & Medium & High & Total \\
\hline Current smoker & 1.6 & 2.0 & 0.1 & $3.7(26.1)$ \\
Ex-smoker & 0.9 & 1.4 & 0.3 & $2.6(18.3)$ \\
Never smoked & 2.8 & 4.6 & 0.5 & $7.9(55.6)$ \\
Total & 4.2 & 4.1 & 2.4 & 10.7 \\
\hline
\end{tabular}

Values in parentheses are percentages of total deaths

Table 9. Cancer risk per 10000 person-years

\begin{tabular}{lccc}
\hline & \multicolumn{3}{c}{ Fitness category } \\
\cline { 2 - 4 } & Low & Medium & High \\
\hline Men & & & \\
Women & 12.6 & 4.8 & 3.7 \\
Men + women & 11.5 & 7.6 & 0.9 \\
& 12.3 & 5.5 & 3.1 \\
\hline
\end{tabular}

that associated with smoking) is substantially reduced on moving from the lowest fitness quintile to the next two quintiles and, due mainly to the influence of the female subjects, there is a further smaller advantage in moving to the upper two fitness quintiles.

\section{Discussion}

The present data support the inference of Blair et al. ${ }^{3}$ that there is an association between fitness and the subsequent death rate from cancer, although after eliminating effects due to the smoking/low fitness association, the effect seems substantially smaller than suggested in the original paper. Moreover, there is no evidence to indicate whether the association with fitness is causal in nature, or is attributable to a mutual association with some other unmeasured variable.

There remain several additional caveats to the analysis. First, the follow-up reached a commendable $95 \%$ of participants, but it was not possible to account for a substantial 667 individuals, despite an exhaustive search. It is a reasonable supposition that a proportion of these - perhaps a half - were dead ( 255 men and 78 women). Assuming further no selective difficulty in tracing cancer deaths, some 68 men and 33 women who died of cancer would have been missed, a disturbing $55 \%$ of total cancer deaths. It is probable that there would have been selective difficulty in tracing participants in the lower socioeconomic categories, but such individuals tend to have a low fitness status ${ }^{5}$. It is thus unlikely that a failure to trace all deaths could explain the preponderance of cancer deaths among unfit individuals.

In approximating the matrices for cancer deaths that were not related to smoking, it has been assumed that smoking led to a uniform doubling of cancer risk. However, there may well have been a higher cigarette consumption and thus a greater risk of cancer in the unfit smokers than in smokers with a better treadmill performance. Thus, the procedures adopted may not fully have eliminated the potential impact of smoking upon the association between initial fitness status and cancer rates.

Fitness data were based on treadmill measurements made at entry to a preventive health programme. Those who began the programme with an above average treadmill score may thus have had other good health habits that were not allowed for in this analysis ${ }^{4}$. Moreover, although all subjects were ostensibly in good health at entry, and participants were followed for an average of 8 years, it remains possible that subclinical disease may have limited the exercise tolerance of those who were in the process of developing a tumour at entry. Although Blair et al. ${ }^{3}$ do not provide precise fitness data in their study, it appears from the context that even those subjects falling into the medium fitness category were relatively unfit, probably having a maximal oxygen intake of about $30 \mathrm{ml} \mathrm{kg}^{1} \mathrm{~min}^{-1}$. If further research supports a causal association between poor fitness and cancer rates, protection would thus be associated 
with moving from a very sedentary state (maximal oxygen intake $\left(<21 \mathrm{ml} \mathrm{kg}^{-1} \mathrm{~min}^{-1}\right)$ to what is still a sedentary lifestyle $\left(30 \mathrm{ml} \mathrm{kg}^{-1} \mathrm{~min}^{-1}\right)$. Over this range, the relevant issue may be the proportion of body fat or a genetic difference of body build rather than the pattern of personal physical activity, and any causal explanation could not be clearly linked to earlier studies that have shown protection against colonic or reproductive cancer from athletic participation or engagement in a demanding physical occupation. It may be for this reason that Blair et al. ${ }^{3}$ found a stronger association between physical fitness and overall cancer mortality rates than might have been anticipated from some previous studies ${ }^{1,2}$.

\section{References}

1 Shephard RJ. Exercise and malignancy. Sports Med 1986; 3: 235-41.

2 Shephard RJ. Physical activity and cancer. Int J Sports Med 1990; 11: 413-20.

3 Blair SN, Kohl HW, Paffenbarger RW, Clark DG et al. Physical fitness and all-cause mortality. A prospective study of healthy men and women. JAMA 1989; 262: 2395-401.

4 Shephard RJ. Exercise and lifestyle change. $\mathrm{Br} J$ Sports Med 1989; 23: 11-22.

5 Fitness Canada. Fitness and Lifestyle in Canada. Ottawa: Fitness and Lifestyle Research Institute, 1983.

\section{Erratum}

Jeffery RS, Caiach S. Waterbike injuries. Br J Sports Med 1991; 25: 232-4.

The publishers wish to apologise for a printing error which occurred in the above article. Figure 4 was reversed left to right but is shown here in the correct orientation.

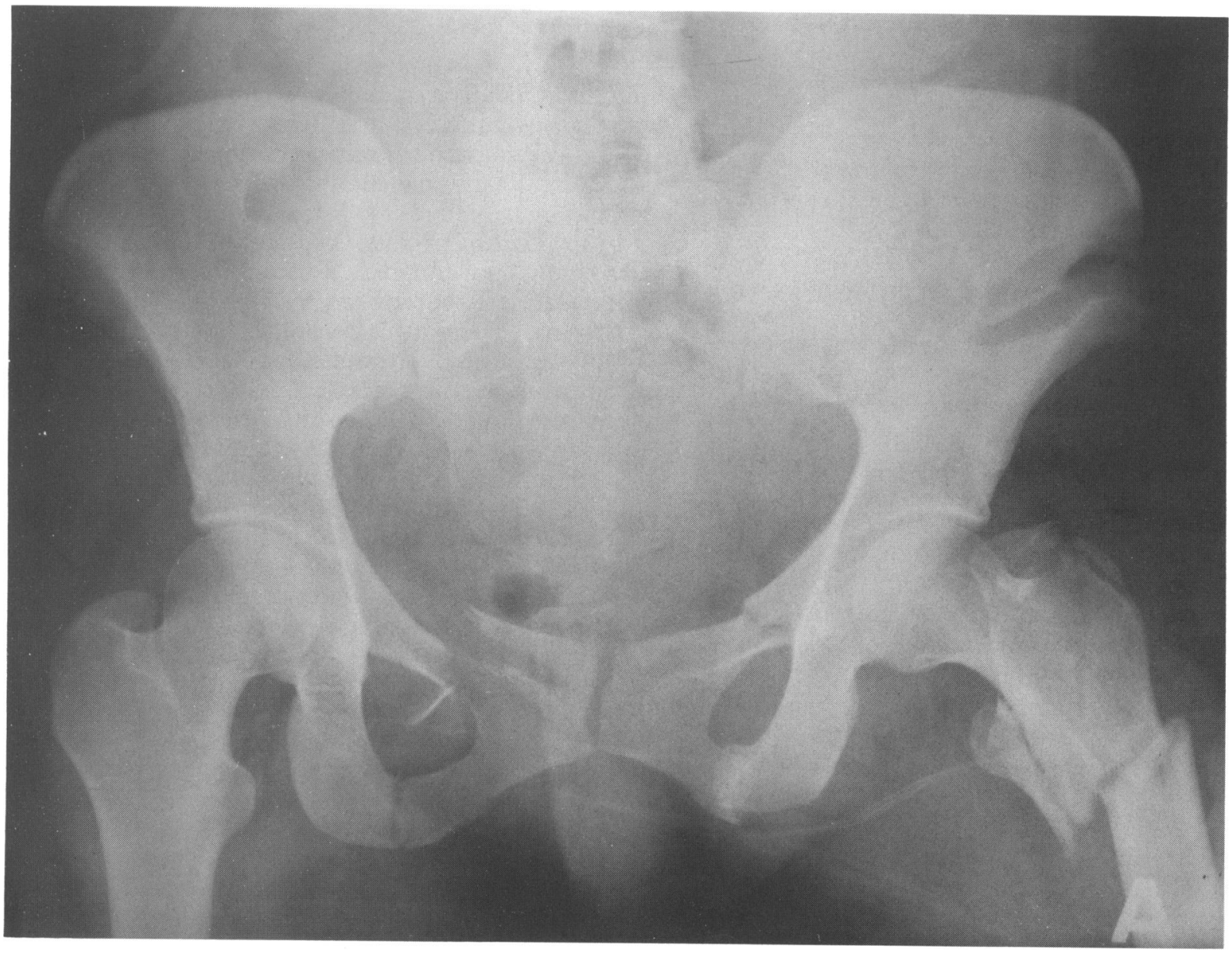

Figure 4. Case 6: pelvic and left hip injury 